\title{
Surface states on a topologically nontrivial semimetal: The case of $\mathrm{Sb}(110)$
}

\author{
Marco Bianchi, ${ }^{1}$ Dandan Guan, ${ }^{1,2}$ Anna Stróżecka,${ }^{3}$ Celia H. Voetmann, ${ }^{1}$ Shining Bao, ${ }^{2}$ Jose Ignacio Pascual, ${ }^{3}$ \\ Asier Eiguren, ${ }^{4,5}$ and Philip Hofmann ${ }^{1}$ \\ ${ }^{1}$ Department of Physics and Astronomy, Interdisciplinary Nanoscience Center, Aarhus University, 8000 Aarhus C, Denmark \\ ${ }^{2}$ Department of Physics, Zhejiang University, Hangzhou, 310027 China \\ ${ }^{3}$ Institut für Experimentalphysik, Freie Universität Berlin, 14195 Berlin, Germany \\ ${ }^{4}$ Departameto de Física de la Materia Condensada, EHU/UPV, Barrio Sarriena sn 48940 Leioa, Spain \\ ${ }^{5}$ Donostia International Physics Center (DIPC), Paseo Manuel de Lardizabal, 4. 20018 Donostia-San Sebastian, Spain
}

(Received 9 February 2012; revised manuscript received 24 March 2012; published 16 April 2012)

\begin{abstract}
The electronic structure of $\mathrm{Sb}(110)$ is studied by angle-resolved photoemission spectroscopy and first-principles calculations, revealing several electronic surface states in the projected bulk band gaps around the Fermi energy. The dispersion of the states can be interpreted in terms of a strong spin-orbit splitting. The bulk band structure of $\mathrm{Sb}$ has the characteristics of a strong topological insulator with a $\mathbb{Z}_{2}$ invariant $v_{0}=1$. This puts constraints on the existence of metallic surface states and the expected topology of the surface Fermi contour. However, bulk Sb is a semimetal, not an insulator, and these constraints are therefore partly relaxed. This relation of bulk topology and expected surface-state dispersion for semimetals is discussed.
\end{abstract}

DOI: 10.1103/PhysRevB.85.155431

PACS number(s): 73.20.At, 71.10.Ca, 79.60.Bm

\section{INTRODUCTION}

Topological insulators are a recently discovered class of materials with fascinating properties: while the inside of the solid is insulating, fundamental topological considerations require the surfaces to be metallic. ${ }^{1-6}$ The metallic surface states show an unconventional spin texture ${ }^{7,8}$ and electron dynamics. $^{9-11}$ They are furthermore stable in the sense that their existence is a bulk property and derived from the bulk electronic structure. The surface-state spectrum can be predicted by the single $\mathbb{Z}_{2}$ invariant $v_{0}$. For $v_{0}=1$, the bulk electronic structure is that of a strong topological insulator and gapless, stable surface states are expected, whereas this is not the case for $v_{0}=0 .^{1,2}$

The topology of the bulk bands does not only permit the prediction of metallic surface states, it also puts rigorous constraints on the number of the bands crossing the Fermi energy in certain high-symmetry directions, and even on the number of closed Fermi contours encircling high-symmetry points. ${ }^{1,2}$ These topological predictions were found to be obeyed for the (111) surface of the three-dimensional topological insulators $\mathrm{Bi}_{1-x} \mathrm{Sb}_{x}(0.09<x<0.18),{ }^{1,4,7}$ as well as $\mathrm{Bi}_{2} \mathrm{Se}_{3}$ and $\mathrm{Bi}_{2} \mathrm{Te}_{3}{ }^{12-16}$

The surface electronic structure of the group- $\mathrm{V}$ semimetals bismuth and antimony is very similar to that of the topological insulator $\mathrm{Bi}_{1-x} \mathrm{Sb}_{x}$. The similarity to the corresponding $\mathrm{Bi}$ surfaces is not surprising since $x$ is quite small. Indeed, all Bi surfaces studied so far have been found to support metallic electronic states, in contrast to the semimetallic bulk. ${ }^{17-20}$ It has been suggested ${ }^{18}$ and later shown that these surface states are split by the spin-orbit interaction, ${ }^{21}$ and the Bi surface states were found to show some characteristics that were later discussed in connection with the topological insulators, such as the absence of backscattering ${ }^{22}$ or the fact that charge density waves can not be formed even for nested Fermi contours. ${ }^{23}$ The similarity of $\mathrm{Bi}_{1-x} \mathrm{Sb}_{x}$ to pure $\mathrm{Sb}$ is that the gapped alloy inherits its topological character from $\mathrm{Sb}\left(v_{0}=1\right)$, not from $\mathrm{Bi}\left(v_{0}=0\right)$. Thus, $\mathrm{Sb}$ has the characteristics of a strong topological insulator while it is a semimetal, not an insulator.
Experimental electronic-structure results have so far only been reported for the $\mathrm{Sb}(111)$ surface. ${ }^{7,24}$

In this paper, we present results from the electronic structure of $\mathrm{Sb}(110)$. This surface is interesting for two reasons. The first is the nontrivial bulk topology of Sb. The second is that, compared to to the (111) surface, (110) has more distinct socalled time-reversal-invariant momenta (four instead of two) and this provides the opportunity to study the surface-state topology in more detail.

An interesting question is what the bulk topological considerations imply for the semimetal surfaces. Strictly speaking, there is no fundamental reason to expect metallic surface states on $\mathrm{Bi}$ or $\mathrm{Sb}$, but such states have so far always been found and appear to be quite robust. For $\mathrm{Sb}(110)$, we argue that while the semimetallic character of the substrate inhibits a statement on the global existence of surface states, the dispersion of states in certain high-symmetry directions of $k$ space (directions without bulk projected states) can still be rigorously compared to topological predictions.

Figure 1 provides an overview of the $\mathrm{Sb}(110)$ surface in real and reciprocal space. Figures 1(a) and 1(b) show a model of the truncated bulk surface and the scanning tunneling microscopy (STM) topography, ${ }^{25}$ respectively. Neither STM nor low-energy diffraction give any indication of a surface reconstruction. Figure 1(c) illustrates the bulk Brillouin zone of $\mathrm{Sb}$ with the bulk Fermi surface and the projection of the former onto the (110) surface. A remarkable feature of the $\mathrm{Sb}(110)$ surface is its low symmetry with a mirror line being the only symmetry element. Nevertheless, time-reversal symmetry guarantees the electronic structure for high-symmetry points such as $\bar{M}$ to be the same for all four equivalent points, ${ }^{18}$ effectively giving rise to a second mirror symmetry in the observed band dispersion.

\section{EXPERIMENTAL AND THEORETICAL METHODS}

We have investigated the surface electronic structure of $\mathrm{Sb}(110)$ using angle-resolved photoemission spectroscopy 
(a)

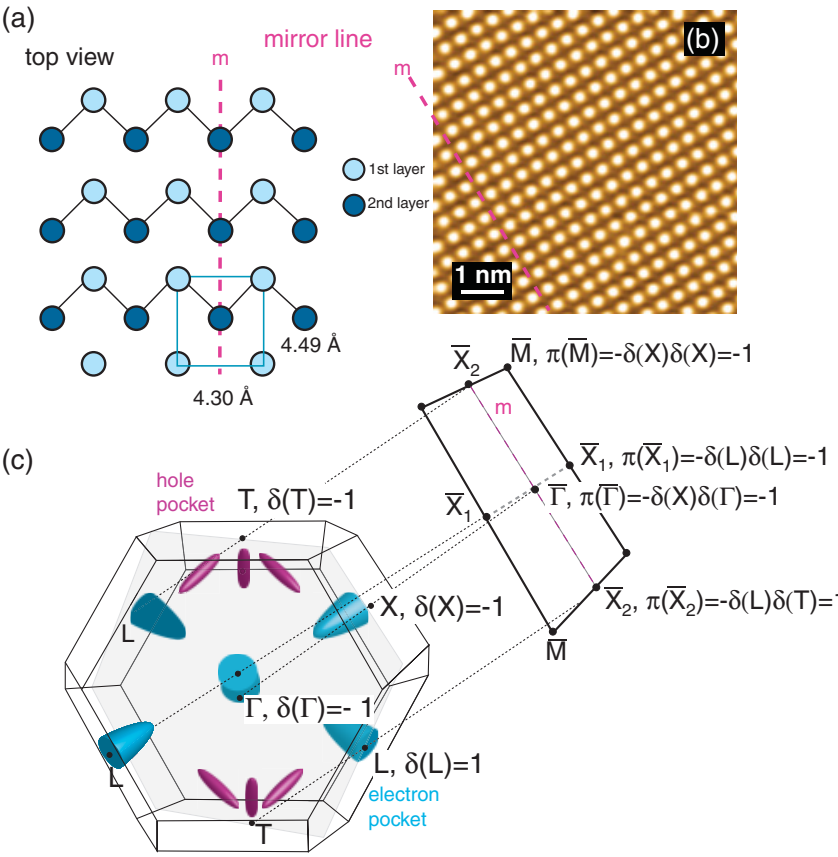

FIG. 1. (Color online) (a) Truncated-bulk geometric structure of $\mathrm{Sb}$ (110). (b) STM topography of the surface. (c) Bulk Brillouin zone of $\mathrm{Sb}$ with a sketch of the Fermi-surface elements (qualitatively and not to scale), together with a projection onto the $\mathrm{Sb}(110)$ surface Brillouin zone. The gray plane is a bulk mirror plane which projects onto a surface mirror line. For the eight bulk time-reversal-invariant momenta (TRIMs) $\Gamma_{i}$, the parity invariants $\delta\left(\Gamma_{i}\right)$ are given. These are projected onto the four surface TRIMs $\lambda_{a}$, resulting in the surface fermion parity $\pi\left(\lambda_{a}\right)=-\delta\left(\Gamma_{i}\right) \delta\left(\Gamma_{j}\right)$. The resulting values of $\pi\left(\lambda_{a}\right)$ are denoted at the surface TRIMs (Ref. 26).

(ARPES). Data were taken at the SGM-III beamline of the synchrotron radiation facility ASTRID in Aarhus. ${ }^{27}$ The combined energy and angular resolution were better than $10 \mathrm{meV}$ and $0.13^{\circ}$, respectively. The data shown here were all taken using a photon energy of $20 \mathrm{eV}$. The $\mathrm{Sb}(110)$ surface was cleaned in situ by cycles of sputtering and annealing to $520 \mathrm{~K}$. The clean surface showed a $(1 \times 1)$ low-energy electron diffraction pattern with the only symmetry being the mirror line that defines the $\bar{\Gamma} \bar{X}_{2}$ direction of surface Brillouin zone. ${ }^{28}$ During the photoemission measurements, the sample was kept at a temperature of $60 \mathrm{~K}$. We have also studied the surface topology using scanning tunneling microscopy at $5 \mathrm{~K}$.

The $\mathrm{Sb}(110)$ surface was modeled considering a repeated slab system consisting of 54 layers, relaxed up to forces $<10^{-4}$ Ry/a.u. We used fully relativistic norm-conserving pseudopotentials as described in Ref. 29, with the energy cutoff corresponding to $E_{c}=60 \mathrm{Ry}$. We considered the Perdew-Burke-Ernzerhof implementation ${ }^{30}$ of the generalized gradient approximation within a noncollinear implementation of density functional theory (DFT). ${ }^{29,31}$ The self-consistent DFT cycle was completed with a $20 \times 20$ Monkhorst-Pack mesh. In order to calculate the projection of the bulk band structure onto the (110) surface, we have also used the tight-binding scheme of Liu and Allen. ${ }^{32}$ This is expected to give reliable results very close to the Fermi energy since the tight-binding parameters have been optimized to reproduce the bulk Fermi surface. While we find the projected band structure and Fermi surface of the DFT calculation to be consistent with the tight-binding result, very small errors in such a calculation or merely the limited slab size can qualitatively change the projected Fermi surface in a semimetal where the electron and hole pockets of the Fermi surface are less than $100 \mathrm{meV}$ deep.

\section{RESULTS}

The results of the ARPES investigation are shown in Figs. 2 and 3. The figures show the photoemission intensity at the Fermi level and as a function of binding energy along some high-symmetry directions, respectively. For clarity, both figures show two versions of the data: only the measured intensity and this intensity superimposed with colored lines to guide the eye for identifying the surface states and the projected bulk band structure. Note that the colored lines are only drawn where the data show clearly identifiable structures; they are not representations of the actual Fermi contour or dispersion that is expected to continue even if the colored lines end.

The Fermi contour in Fig. 2 shows several surface-related features, identified by their location outside the projected band continuum and the fact that their position is insensitive to the photon energy used. Most pronounced are a circular contour around $\bar{M}$ (outlined in red) and a butterflylike feature that encloses the $\bar{X}_{1}$ point (blue). Two smaller pockets are seen along the $\bar{M} \bar{X}_{2}$ line (light blue) and the $\bar{X}_{2} \bar{\Gamma}$ line (green). The latter falls partly into the bulk continuum and thus has the character of a surface resonance there. Finally, we find a faint trace split off the "butterfly wing" and dispersing toward $\bar{X}_{2}$ (magenta) and some faint intensity crossing the $\bar{X}_{2} \bar{\Gamma}$ line (also magenta). As we shall see later from the calculated electronic structures, these features are probably joint to form a large hole pocket around $\bar{\Gamma}$, but this is not clearly seen in the data.

The detailed character of the features emerges from the dispersion shown in Fig. 3. The circular contour around $\bar{M}$ (red) is a hole pocket, whereas the feature along $\bar{M} \bar{X}_{2}$ (light blue) is a shallow electron pocket. The two features can be interpreted as spin-orbit split partners stemming from the

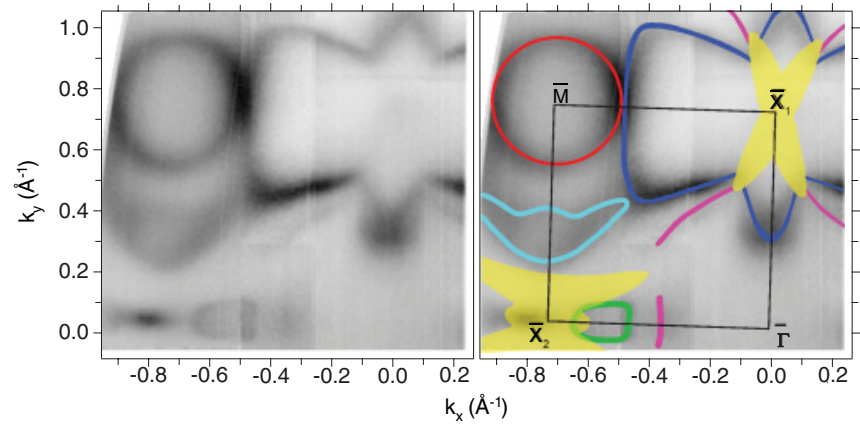

FIG. 2. (Color online) Photoemission intensity at the Fermi level. Dark corresponds to high intensity and the dark features outside the projected bulk band continuum are interpreted as the surface Fermi contour. The left part of the figure shows the raw data, whereas the different structures are indicated by colored lines as a guide to the eye on the right part. Different colors are used for different surface-state features. The light yellow areas correspond to the projected bulk Fermi surface (states within $\pm 5 \mathrm{meV}$ of the Fermi energy), calculated using the tight-binding parameters from Liu and Allen (Ref. 32). 


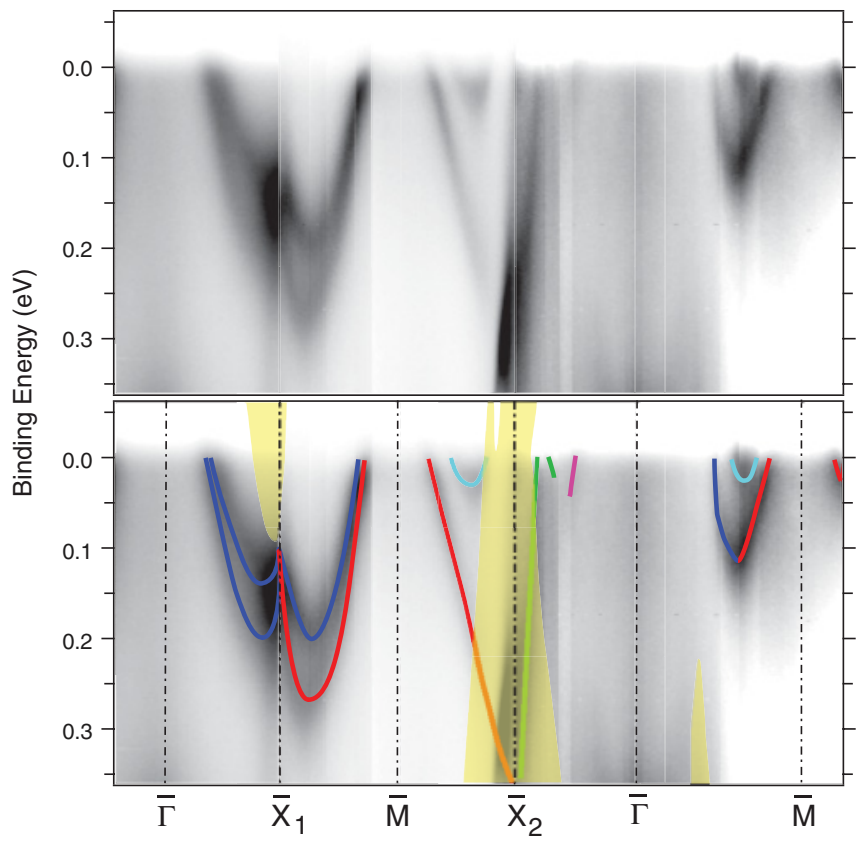

FIG. 3. (Color online) Photoemission intensity as a function of binding energy along different directions of the surface Brillouin zone. The upper part shows the raw data. In the lower part, the observed bands are emphasized by colored lines. The colors are corresponding to those used in Fig. 2. The light yellow areas represent the projected bulk band structure.

same state. The state is unoccupied and assumed to be spin degenerate at $\bar{M}$, but split away from this point. One of the split bands disperses steeply downward and forms the hole pocket. The other one forms the electron pocket along $\bar{M} \bar{X}_{2}$. At $\bar{X}_{1}$, the surface state is also twofold degenerate but it is occupied and can thus be observed by ARPES. Away from $\bar{X}_{1}$, the state clearly splits into two bands, both along $\bar{X}_{1} \bar{M}$ and $\bar{X}_{1} \bar{\Gamma}$, but these bands merge again and are too close to be distinguished at the Fermi level crossings along these high-symmetry directions. Consequently, the Fermi level crossings along $\bar{X}_{1} \bar{\Gamma}$ and $\bar{X}_{1} \bar{M}$ are double crossings. Close to the $\bar{X}_{1} \bar{M}$ direction, the two bands forming the double crossing separate into the circle and the butterfly.

The weakest feature in the data is the band which splits off from the butterfly structure and disperses toward $\bar{X}_{2}$ (magenta). Its presence is clearly required by the overall Fermi contour topology: the two spin-split surface-state branches are occupied at $\bar{X}_{1}$ and empty at $\bar{M}$ and $\bar{\Gamma}$. Consequently, two Fermi level crossings have to be found along the corresponding high-symmetry lines. Along the $\bar{X}_{1} \bar{M}$ direction, the two crossings are formed by the circular contour around $\bar{M}$ and the "wing" of the butterfly, which is nondegenerate. Along $\bar{X}_{1} \bar{\Gamma}$, the blue feature is twofold degenerate. The weak magenta feature corresponds to the second Fermi level crossing. As it disperses away from the butterfly, its intensity diminishes so much that it can not be established whether it continues to the $\bar{X}_{2} \bar{\Gamma}$ line and merges with the other magenta feature observed there, or if it merges into the projected bulk bands close to $\bar{X}_{2}$.

The identification of the electronic structure near the $\bar{X}_{2} \bar{\Gamma}$ line is more difficult due to the presence of bulk states. As
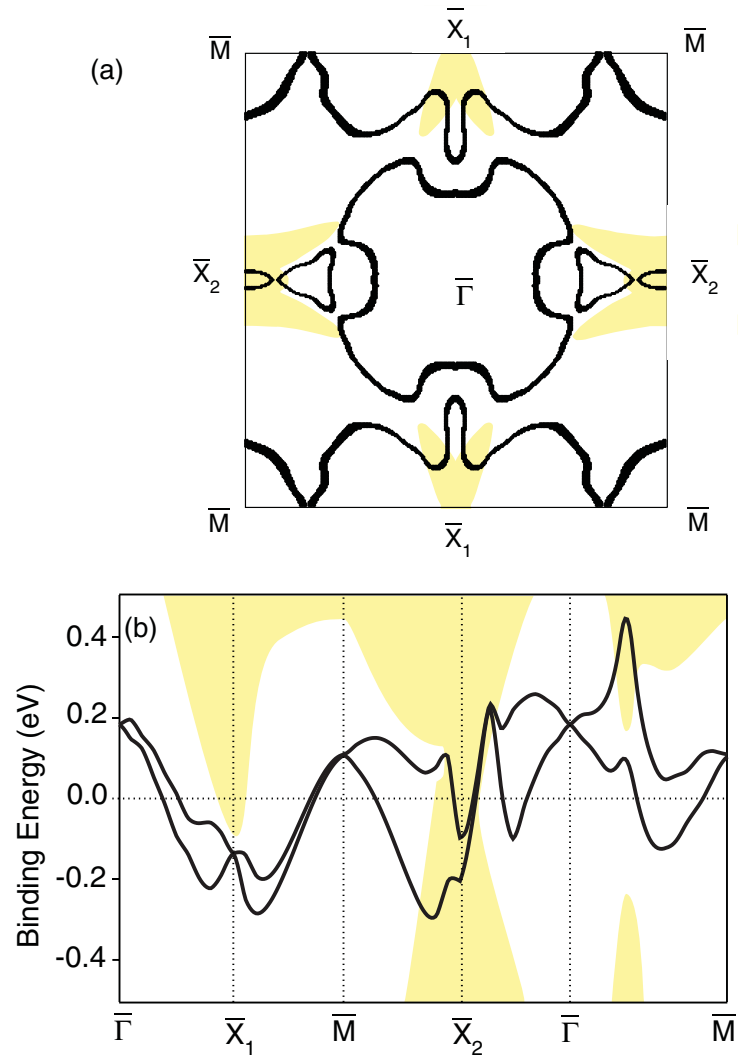

FIG. 4. (Color online) (a) Calculated Fermi contour and (b) surface-state dispersion. The lines represent the surface states from the DFT slab calculation. The yellow continuum is the projected bulk band structure calculated using the tight-binding parameters from Liu and Allen (Ref. 32).

pointed out above, the feature outlined in green appears to be a closed pocket around this line, but it falls partly into the bulk continuum. Also, only the band giving rise to the crossing nearest to $\bar{X}_{2}$ is clearly identifiable in the dispersion, the crossing further away from $\bar{X}_{2}$ is very weak, and the dispersion can not be followed to higher binding energies. Nevertheless, the dispersion of the first band suggests that the pocket is a hole pocket. The magenta feature is well separated from the bulk continuum at the Fermi level, but it is very broad and its dispersion is only clearly observed near $E_{F}$. The sign of its group velocity would be consistent with the feature being part of a hole pocket around $\bar{\Gamma}$.

The calculated Fermi contour and surface-state dispersion are given in Fig. 4. Overall, a very good agreement with the experimental findings is obtained. The main features of the measured Fermi contour are immediately recognized, especially the butterfly close to $\bar{X}_{1}$ and the hole pocket around $\bar{M}$. The calculation also confirms the interpretation of the surface states as being nondegenerate spin-split bands that are degenerate only at points where this degeneracy is enforced by symmetry (so-called time-reversal-invariant momenta, see below).

Some smaller details are not entirely captured by the theoretical results. First, the very shallow electron pocket along $\bar{M} \bar{X}_{2}$ appears in the calculations as a dip in the dispersion of the band, which does not cross the Fermi level. This can easily 
be accounted for by small uncertainties in the calculations (see below). In the discussion of the surface-state dispersion's topological properties, it will also become apparent that the topological character of the surface states is not affected by the presence or absence of this electron pocket. The second apparent difference between experiment and theory is the presence of a large hole pocket encircling the $\bar{\Gamma}$ point in the latter. However, a slightly different dispersion in the theory would cause the hole pocket to merge with the butterfly structure, giving rise to the experimentally observed double crossing on the $\bar{\Gamma} \bar{X}_{1}$ line and to the weak structure split off the butterfly when going from this line toward $\bar{M}$. In fact, merging the magenta structures in Fig. 2 would give rise to a large electron pocket around $\bar{\Gamma}$, and it seems likely that the lines should be merged since an open Fermi contour would be unphysical.

The deviation of measured and calculated surface-state energies is usually below $100 \mathrm{meV}$, which is very satisfactory, considering the intrinsic accuracy limits of the employed density functional theory. We also note that many-body effects such as correlations or the electron-phonon coupling are not included in the calculations. These could have an effect on the experimental dispersion close to the Fermi energy through the resulting real part of the self-energy. The absolute magnitude of such effects is not expected to be large for $\mathrm{Sb}$, but effects of the order of several $10 \mathrm{meV}$ are certainly possible and would be noticeable.

As far as the surface-state dispersion is concerned, we can thus draw the following conclusions: the electronic structure of $\mathrm{Sb}(110)$ shows several electronic surface states crossing the Fermi level. It thus has the character of a good metal in contrast to the semimetallic bulk. This appears to be a general feature of the $\mathrm{Bi}$ and $\mathrm{Sb}$ semimetal surfaces and it has been explained as resulting from the combination of symmetry breaking and a strong spin-orbit interaction. ${ }^{18,20,21,24,33}$ The electronic structure of $\mathrm{Sb}(110)$ is similar to that of $\mathrm{Bi}(110)$ in some parts of the surface Brillouin zone. The observed and calculated dispersion is mostly consistent with the expected degeneracy at high-symmetry points (see following) and a spin-orbit splitting away from these points. While we do not directly measure the spin of the bands, this interpretation appears to be based on solid ground. It is consistent with the findings on all other $\mathrm{Bi}, \mathrm{Sb}$, and $\mathrm{Bi}_{1-x} \mathrm{Sb}_{x}$ surfaces and with the calculations reported here. Moreover, the spin-split nature of the states is also confirmed by the absence of the characteristic backscattering standing waves in our STM investigations. ${ }^{34}$ Compared to $\mathrm{Bi}(110)$, the spin-orbit splitting is smaller, as expected. This has the interesting consequence of concentrating the spin-orbit split bands in an even narrower window around the Fermi energy, presumably leading to a higher density of states there, and possibly opening the option of tuning the electronic structure as to purposefully move van Hove singularities to the Fermi level. ${ }^{35,36}$

\section{DISCUSSION}

We now turn back to the more general question of how much topological considerations affect the existence of surface states on semimetal surfaces. We discuss this using the specific example of $\mathrm{Sb}(110)$, but we also address other surface orientations and the case of $\mathrm{Bi}$.

The topological situation for materials with inversion symmetry in general, and $\mathrm{Bi}, \mathrm{Sb}$, and $\mathrm{Bi}_{1-x} \mathrm{Sb}_{x}$ in particular, has been studied in great detail by Teo, Fu, and $\mathrm{Kane}^{26}$ and we summarize some of their results here. The $\mathbb{Z}_{2}$ invariant $v_{0}$, which dictates the topological character of the solid, is given by

$$
(-1)^{\nu_{0}}=\prod_{i=1}^{8} \delta\left(\Gamma_{i}\right),
$$

where the $\delta\left(\Gamma_{i}\right)$ are the parity invariants of the eight bulk timereversal-invariant momenta (TRIMs) $\Gamma_{i}$, defined by $-\Gamma_{i}=$ $\Gamma_{i}+G$, where $G$ is a bulk reciprocal lattice vector. For bulk Sb, the TRIMs are $\Gamma, T, L$, and $X$. Their $\delta\left(\Gamma_{i}\right)$ values are calculated by

$$
\delta\left(\Gamma_{i}\right)=\prod_{n=1} \xi_{2 n}\left(\Gamma_{i}\right),
$$

where the $\xi_{2 n}\left(\Gamma_{i}\right)= \pm 1$ are the parity eigenvalues of the $2 n$th occupied band at $\Gamma_{i}$, obtained from a bulk band-structure calculation. ${ }^{32,37}$ The $\delta\left(\Gamma_{i}\right)$ values for the bulk TRIMs are given in Fig. 1(c). The key difference between $\mathrm{Bi}$ on one hand and $\mathrm{Bi}_{1-x} \mathrm{Sb}_{x}$ or $\mathrm{Sb}$ on the other hand is that $v_{0}=0$ for the former and 1 for the latter. This stems from a single change of a parity invariant $\delta(L)$ from -1 in $\mathrm{Bi}$ to 1 in the other compounds (see Table I in Ref. 26).

The bulk parity invariants can now be used to describe fundamental properties of the surface electronic structure. To do this, the surface TRIMs $\Lambda_{a}$ are defined such that $\Lambda_{a}=$ $-\Lambda_{a}+g$, where $g$ is a surface reciprocal lattice vector. For these points $\left(\bar{\Gamma}, \bar{M}, \bar{X}_{1}\right.$, and $\left.\bar{X}_{2}\right)$, the surface-state bands must be spin degenerate, even in the presence of a strong spinorbit interaction. For each surface TRIM, the so-called surface fermion parity $\pi\left(\Lambda_{a}\right)$ can be determined. Essentially, $\pi\left(\Lambda_{a}\right)$ is obtained by projecting out the bulk parity invariants onto the corresponding surface TRIMs [see Fig. 1(c)], using the relation $\pi\left(\Lambda_{a}\right)=-\delta\left(\Gamma_{i}\right) \delta\left(\Gamma_{j}\right) .{ }^{26}$ For instance, $\pi(\bar{\Gamma})$ has to be calculated from the parity invariants of the bulk $\Gamma$ and $X$ points, which are both -1 and hence $\pi(\bar{\Gamma})=-(-1)^{2}$. As mentioned above, the only difference in bulk parity invariants between $\mathrm{Bi}$ and $\mathrm{Sb}$ is at the $L$ point, where $\delta(L)=-1$ and 1 for $\mathrm{Bi}$ and $\mathrm{Sb}$, respectively, and this difference implies a change the surface fermion parity at $\bar{X}_{2}$ from -1 to 1 .

The surface fermion parity in Fig. 1(c) can be used to predict the number of closed Fermi contours around a surface TRIM or the number of Fermi level crossings between two surface TRIMs. We start with the latter prediction, which is easier to verify experimentally. The number of crossings has to be zero or even if the two surface TRIMs have the same parity and odd otherwise. Based on these rules, Teo, Fu, and $\mathrm{Kane}^{26}$ have made detailed predictions of the qualitative surface electronic structure of many surfaces. We adopt their result for $\mathrm{Bi}_{1-x} \mathrm{Sb}_{x}$ to the topologically identical case of $\mathrm{Sb}(110)$ and find that there must be an odd number of Fermi level crossings between $\bar{X}_{2}$ and any other surface TRIM and an even number between two surface TRIMs not involving $\bar{X}_{2}$.

An inspection of Figs. 2 and 3 shows that this is the case, despite the presence of bulk Fermi-surface projections. The 
situation is clearest for the $\bar{X}_{1}$ point. At $\bar{X}_{1}$, we find the two states to be degenerate, as predicted, and in both the $\bar{X}_{1} \bar{\Gamma}$ and $\bar{X}_{1} \bar{M}$ direction we find two Fermi level crossings, also agreeing with the prediction.

For $\bar{X}_{2}$, the situation is difficult to determine solely on the basis of our experiments because the intensity of the surface bands close to this point is very weak, due to the presence of the bulk band continuum. At first glance, the number of Fermi level crossings seems to be as predicted from the topological arguments: along both directions, $\bar{M} \bar{X}_{2}$ and $\bar{X}_{2} \bar{\Gamma}$, we can identify one closed contour plus an extra crossing, and thus an odd number of crossings.

We can also compare the experimentally observed number of closed Fermi contours around each surface TRIM to the topological predictions based on the surface fermion parity. A surface TRIM with $\pi\left(\Lambda_{a}\right)=-1$ is expected to be encircled by an odd number of Fermi contours, while the number is zero or even for $\pi\left(\Lambda_{a}\right)=1$. Thus, we expect an odd number of contours around $\bar{\Gamma}, \bar{M}$, and $\bar{X}_{1}$ and an even number around $\bar{X}_{2}$. For $\bar{M}$ this is fulfilled, as the point is encircled by one hole pocket. $\bar{X}_{1}$ is also encircled by only one contour, which is consistent with $\pi=1$. For $\bar{\Gamma}$ the situation is unclear, as it depends on the weak feature which is split off the butterfly. It is likely that this feature connects to the observed crossing along the $\bar{X}_{2} \bar{\Gamma}$ line, giving rise to a circular contour around $\bar{\Gamma}$, as also suggested by our calculation. For $\bar{X}_{2}$ the situation is again obscured due to the projected bands around this point and it is difficult to determine whether this state is encircled by any closed Fermi contour.

The situation becomes clearer when we look at the calculated electronic structure and test it against the topological predictions. For $\bar{\Gamma}, \bar{M}$, and $\bar{X}_{1}$ and the lines between these points, the number of closed contours and Fermi level crossings are as expected. For $\bar{X}_{2}$, however, the topological predictions are seemingly violated: the calculations show an additional closed Fermi contour around this point, and thus the number of Fermi level crossings between $\bar{X}_{2}$ and any other TRIM becomes even, not odd as predicted. However, the apparent discrepancy is easily resolved upon noticing that the additional closed contour around $\bar{X}_{2}$ lies entirely within the bulk continuum and the states are thus not surface states anymore. In fact, the calculations find that the surface band is mixed with bulk states and penetrates deeply into the bulk of the slab. This is also responsible for the apparent lifting of the degeneracy at $\bar{X}_{2}$ (thinner slabs give a bigger splitting). The deep penetration of surface bands into the bulk breaks down the validity of surface fermion parity rules and, hence, the predictions of the topological theory, which can only be strictly applied for insulators.

We address this issue in more detail and ask to what degree topological arguments can be used to make firm predictions as to the surface-state dispersion on semimetal surfaces. Naively, one could expect the topological predictions, such as the number of Fermi level crossings between two TRIMS, not to have any significance anymore because they have been derived under the condition that the bulk material is an insulator. Fortunately, this is not quite the case. In fact, we have found that the topological predictions are mostly followed here, as for the other semimetal surfaces studied so far, ${ }^{26}$ and there are reasons why one should expect this.
In a simple picture, the need for an odd number of Fermi level crossings between two surface TRIMs arises because of their different surface fermion parity. In an insulator, due to the existence of a global energy gap around $E_{F}$, the necessary parity change between surface TRIMs can only be achieved by surface states. On a semimetal, on the other hand, a surfaceprojected bulk state can be used for this purpose, if a projected bulk Fermi surface can be found between the two surface TRIMs in question. This argument implies that the number of $E_{F}$ crossings between TRIMs without any projected Fermi surface in-between them should be as topologically predicted. For $\mathrm{Sb}(110)$, the only such connection is between $\bar{\Gamma}$ and $\bar{M}$, and this cut does indeed show an even number of surface Fermi level crossings, as predicted. Similar arguments can be made for the $\bar{X}_{1} \bar{\Gamma}$ and $\bar{X}_{1} \bar{M}$ directions. Here, there is a bulk Fermisurface projection (at $\bar{X}_{1}$ ), but the surface-state dispersion lies completely outside this projection and, consequently, it is also in accordance with the topological predictions. In all these situations, we only observe an even number of Fermi level crossings between two TRIMs and thus a topologically trivial situation.

It is interesting to relate these considerations to the case of $\mathrm{Sb}(111)$. The band topology of this surface has been discussed in great detail in Ref. 7. It was found to be consistent with the predicted result ${ }^{26}$ in the sense that the $\bar{\Gamma}$ point is encircled by a closed Fermi contour, whereas the other TRIM $(\bar{M})$ is not. The number of surface-state Fermi level crossings between $\bar{\Gamma}$ and $\bar{M}$, however, was found to be even (2) instead of the topologically predicted odd number of crossings. The reason why this is possible is that there is a projected hole pocket along the line connecting $\bar{\Gamma}$ and $\bar{M}$, such that a third surface-state crossing is suppressed by a band ending in this projected hole pocket.

Finally, the topological considerations for semimetals would still permit the surface to be metallic for topological reasons, i.e., one could expect topologically protected surfacestate Fermi level crossings. This would require an odd number of crossings between two TRIMS without a projected bulk Fermi-surface element in-between. There is no obvious candidate for such a situation among the low-index surface of $\mathrm{Bi}$ and $\mathrm{Sb}$.

Concluding, we have presented experimental and theoretical results on the electronic structure of $\mathrm{Sb}(110)$, a non-(111) surface of a topologically nontrivial material. Along the directions connecting TRIMs without any bulk Fermi-surface contribution, the observed band dispersion is in excellent agreement with the predictions of the surface bands topology. The topological arguments become invalid if the projected bulk Fermi surface is present and the surface bands are allowed to mix with the bulk states.

\section{ACKNOWLEDGMENTS}

The authors gratefully acknowledge stimulating discussions with C. Kane, H. Dil, and J. Osterwalder, as well as support by the Danish Council for Independent ResearchNatural Sciences, the Deutsche Forschungsgemeinschaft (STR 1151/1-1), and the Spanish Ministry of Science and Innovation (FIS2010-19609-C02-00). 
${ }^{1}$ L. Fu and C. L. Kane, Phys. Rev. B 76, 045302 (2007).

${ }^{2}$ L. Fu, C. L. Kane, and E. J. Mele, Phys. Rev. Lett. 98, 106803 (2007).

${ }^{3}$ J. E. Moore and L. Balents, Phys. Rev. B 75, 121306 (2007).

${ }^{4}$ D. Hsieh, D. Qian, L. Wray, Y. Xia, Y. S. Hor, R. J. Cava, and M. Z. Hasan, Nature (London) 452, 970 (2008).

${ }^{5}$ S. C. Zhang, Physics 1, 6 (2008).

${ }^{6}$ J. E. Moore, Nature (London) 464, 194 (2010).

${ }^{7}$ D. Hsieh, Y. Xia, L. Wray, D. Qian, A. Pal, J. H. Dil, J. Osterwalder, F. Meier, G. Bihlmayer, C. L. Kane et al., Science 323, 919 (2009). ${ }^{8}$ D. Hsieh, Y. Xia, D. Qian, L. Wray, F. Meier, J. H. Dil, J. Osterwalder, L. Patthey, A. V. Fedorov, H. Lin et al., Phys. Rev. Lett. 103, 146401 (2009).

${ }^{9}$ M. Konig, S. Wiedmann, C. Brune, A. Roth, H. Buhmann, L. W. Molenkamp, X.-L. Qi, and S.-C. Zhang, Science 318, 766 (2007).

${ }^{10}$ P. Roushan, J. Seo, C. V. Parker, Y. S. Hor, D. Hsieh, D. Qian, A. Richardella, M. Z. Hasan, R. J. Cava, and A. Yazdani, Nature (London) 460, 1106 (2009).

${ }^{11}$ Z. Alpichshev, J. G. Analytis, J.-H. Chu, I. R. Fisher, Y. L. Chen, Z. X. Shen, A. Fang, and A. Kapitulnik, Phys. Rev. Lett. 104, 016401 (2010).

${ }^{12}$ H. Zhang, C.-X. Liu, X.-L. Qi, X. Dai, Z. Fang, and S.-C. Zhang, Nat. Phys. 5, 438 (2009).

${ }^{13}$ Y. Xia, D. Qian, D. Hsieh, L. Wray, A. Pal, H. Lin, A. Bansil, D. Grauer, Y. S. Hor, R. J. Cava et al., Nat. Phys. 5, 398 (2009).

${ }^{14}$ H.-J. Noh, H. Koh, S.-J. Oh, J.-H. Park, H.-D. Kim, J. D. Rameau, T. Valla, T. E. Kidd, P. D. Johnson, Y. Hu et al., Europhys. Lett. 81, 57006 (2008).

${ }^{15}$ Y. L. Chen, J. G. Analytis, J. H. Chu, Z. K. Liu, S. K. Mo, X. L. Qi, H. J. Zhang, D. H. Lu, X. Dai, Z. Fang et al., Science 325, 178 (2009).

${ }^{16}$ D. Hsieh, Y. Xia, D. Qian, L. Wray, J. H. Dil, F. Meier, J. Osterwalder, L. Patthey, J. G. Checkelsky, N. P. Ong et al., Nature (London) 460, 1101 (2009).

${ }^{17}$ C. R. Ast and H. Höchst, Phys. Rev. Lett. 87, 177602 (2001).

${ }^{18}$ S. Agergaard, C. Søndergaard, H. Li, M. B. Nielsen, S. V. Hoffmann, Z. Li, and Ph. Hofmann, New J. Phys. 3, 15.1 (2001).

${ }^{19} \mathrm{Ph}$. Hofmann, J. E. Gayone, G. Bihlmayer, Y. M. Koroteev, and E. V. Chulkov, Phys. Rev. B 71, 195413 (2005).
${ }^{20}$ J. W. Wells, J. H. Dil, F. Meier, J. Lobo-Checa, V. N. Petrov, J. Osterwalder, M. M. Ugeda, I. Fernandez-Torrente, J. I. Pascual, E. D. L. Rienks et al., Phys. Rev. Lett. 102, 096802 (2009).

${ }^{21}$ Y. M. Koroteev, G. Bihlmayer, J. E. Gayone, E. V. Chulkov, S. Blügel, P. M. Echenique, and Ph. Hofmann, Phys. Rev. Lett. 93, 046403 (2004).

${ }^{22}$ J. I. Pascual, G. Bihlmayer, Y. M. Koroteev, H. P. Rust, G. Ceballos, M. Hansmann, K. Horn, E. V. Chulkov, S. Blugel, P. M. Echenique et al., Phys. Rev. Lett. 93, 196802 (2004).

${ }^{23}$ T. K. Kim, J. Wells, C. Kirkegaard, Z. Li, S. V. Hoffmann, J. E. Gayone, I. Fernandez-Torrente, P. Haberle, J. I. Pascual, K. T. Moore et al., Phys. Rev. B 72, 085440 (2005).

${ }^{24}$ K. Sugawara, T. Sato, S. Souma, T. Takahashi, M. Arai, and T. Sasaki, Phys. Rev. Lett. 96, 046411 (2006).

${ }^{25}$ I. Horcas, R. Fernandez, J. M. Gomez-Rodriguez, J. Colchero, J. Gomez-Herrero, and A. M. Baro, Rev. Sci. Instrum. 78, 013705 (2007).

${ }^{26}$ J. C. Y. Teo, L. Fu, and C. L. Kane, Phys. Rev. B 78, 045426 (2008).

${ }^{27}$ S. V. Hoffmann, C. Søndergaard, C. Schultz, Z. Li, and Ph. Hofmann, Nucl. Instrum. Methods Phys. Res., Sect. A 523, 441 (2004).

${ }^{28}$ J. Sun, A. Mikkelsen, M. Fuglsang Jensen, Y. M. Koroteev, G. Bihlmayer, E. V. Chulkov, D. L. Adams, P. Hofmann, and K. Pohl, Phys. Rev. B 74, 245406 (2006).

${ }^{29}$ A. Dal Corso and A. Mosca Conte, Phys. Rev. B 71, 115106 (2005).

${ }^{30}$ J. P. Perdew, K. Burke, and M. Ernzerhof, Phys. Rev. Lett. 77, 3865 (1996).

${ }^{31}$ [www.pwscf.org].

${ }^{32}$ Y. Liu and R. E. Allen, Phys. Rev. B 52, 1566 (1995).

${ }^{33} \mathrm{Ph}$. Hofmann, Prog. Surf. Sci. 81, 191 (2006).

${ }^{34}$ A. Stróżecka et al. (unpublished).

${ }^{35}$ C. R. Ast, J. Henk, A. Ernst, L. Moreschini, M. C. Falub, D. Pacile, P. Bruno, K. Kern, and M. Grioni, Phys. Rev. Lett. 98, 186807 (2007).

${ }^{36}$ C. R. Ast, D. Pacile, L. Moreschini, M. C. Falub, M. Papagno, K. Kern, M. Grioni, J. Henk, A. Ernst, S. Ostanin et al., Phys. Rev. B 77, 081407 (2008).

${ }^{37}$ L. M. Falicov and P. J. Lin, Phys. Rev. 141, 562 (1966). 